\title{
DE LA TEXTURE DES DIÉGÈSES DES ÉCRIVAINES DE LA POSTCOLONIE À LA CONTEXTURE D'UNE MONDIALISATION ALTERNATIVE
}

\author{
Pierre Suzanne Eyenga Onana ${ }^{1}$
}

\begin{abstract}
Résumé: Le choix porté sur le roman d'Anne-Marie Adiaffi, d'Angéline Solange Bonono et celui d'Edwidge Danticat pour conduire notre argumentation n'est pas fortuit. Il permet de mieux visualiser les relations intertextuelles qui lient une écrivaine ivoirienne et une romancière camerounaise et une auteure haïtienne dont les œuvres, pourtant, sont distantes de vingt-trois ans. L'une comme l'autre aborde la question des mariages pré-arrangés ou de la violence dans une perspective constructionniste qui remet au jour les affres de la société patriarcale qui caractérise à ce jour une partie de l'Afrique et maintient la femme dans la posture de "sexe faible». Selon une double perspective critique se réclamant de l'approche genre et du comparatisme, il s'agit de scruter les modes de déclinaison de la mondialisation culturelle entre la Côte d'Ivoire, le Cameroun, Haïti et le monde, par-delà le socle linguistique et géographique qui oppose ces pays. Au regard des passerelles sémantiques que dégage finalement l'herméneutique desdites écrivaines, l'étude s'achève par la monstration des stratégies de restitution de la place de l'écrivaine comme actrice du vivre ensemble et militante féministe de la non-marginalisation de la femme dans la littérature mondiale.
\end{abstract}

Mots clés: écrivaine africaine, intertextualité, constructionnisme, militante féministe

\section{DA TEXTURA DAS DIEGESES DOS ESCRITORES POS- COLONIAIS AO CONTEXTO DE UMA GLOBALIZAÇÃO ALTERNATIVA}

Resumo: A escolha do romance de Anne-Marie Adiaffi, Angéline Solange Bonono e Edwidge Danticat para conduzir nosso argumento não é fortuita. Permite-nos visualizar melhor as relações intertextuais entre um escritor marfinense e um romancista camaronês e um autor haitiano cujas obras, no entanto, têm vinte e três anos de diferença. Ambos abordam a questão dos casamentos ou da violência pré-estabelecidos a partir de uma perspectiva construcionista que expõe os horrores da sociedade patriarcal que caracteriza parte da África até à

\footnotetext{
1 "Maître de conférences" no Departamento de Literatura e Civilização Africanas, na Faculdade de Artes, Letras e Ciências Humanas da Universidade de Yaoundé (Camarões). Pesquisador das modelizações literárias do "viver junto" nas literaturas africanas e da diáspora negra, a epistemologia da literatura (semiologia do texto literário africano e euro-americano escrito), e as questões de feminismos no Gender Studies. Endereço eletrônico: eyonapiers@gmail.com
} 
data e mantém as mulheres na postura de "sexo fraco". A partir de uma dupla perspectiva crítica baseada em uma abordagem de gênero e comparativa, tratase de examinar os modos de declinação da globalização cultural entre Costa do Marfim, Camarões, Haiti e o mundo, além da base linguística e geográfica que se opõe a esses países. Com relação às pontes semânticas que finalmente emergem da hermenêutica dessas escritoras, o estudo termina com a demonstração de estratégias para restaurar o lugar da escritora como atriz de convivência e ativista feminista para a não marginalização das mulheres na literatura mundial.

Palavras-chave: Escritora africana; Intertextualidade; Construcionismo; Ativista feminista.

\section{Introduction}

La conception très diversifiée de la lutte féministe en Afrique ne permet pas d'inscrire le combat des femmes dans la même dynamique revendicative que leurs consœurs des autres continents, notamment européen et américain. A cet égard, la femme musulmane ivoirienne n'aura pas les mêmes préoccupations existentielles que la femme non musulmane camerounaise et vice versa. Subvertissant cet état de choses, Pierrette Herzberger Fofana avalise l'idée que "le féminisme n'a pas été l'une des préoccupations majeures du monde africain » (2000, p. 7). C'est dire que la diversité relevée dans les orientations que revêtent la lutte des femmes pour le respect de leurs droits semble se répercuter dans les postures théoriques assumée par Simone de Beauvoir et Annie Leclerc par exemple. S’il est vrai que les deux postures se joignent globalement à l'argument de Judith Butler (1972) pour montrer que le genre est dans le trouble, force est d'affirmer que pour De Beauvoir, seul prédomine le combat pour l'avènement d'un monde construit. Elle affirme dans cette optique qu'« on ne naît pas femme, on le devient » (1949, p. 285). L'essence de la femme que subliment les partisans du phallocentrisme africain trouve dans ce postulat une incitation à outrepasser les préceptes régissant la culture africaine traditionnelle qui établit la femme comme « un être né à genoux aux pieds de l'homme » (BEYALA, 1995, p. 11).

Avec Annie Leclerc par contre, que ce soit dans Parole de femme (1974) ou dans Hommes et femmes (1985), l'option pour un combat féministe plus modéré s'affiche voire s'impose, tant sa trajectoire féministe œuvre à la promotion d'une femme attachée à la féminité. La femme y est scénarisée, 
DE LA TEXTURE DES DIÉGĖSES DES ÉCRIVAINES DE LA POSTCOLONIE À LA CONTEXTURE D'UNE MONDIALISATION ALTERNATIVE

jouissant des bienfaits de la sexualité (que réglemente par trop Simone de Beauvoir), de la maternité, de l'allaitement maternel, et d'un ensemble de tâches maternelles considérées comme autant de valeurs féminines qui subliment la féminité et encensent celles qui les incarnent. Cette variante féministe s'avère proche de l'androcentrisme prôné par certains traditionalistes africains tel que cela apparaît chez Adiaffi, Bonono ou chez Danticat. Elle se singularise cependant de l'androcentrisme en ceci qu'elle est dépouillée de toute forme de violence débouchant sur l'ostracisation de la femme.

Si l'écrivaine américaine ou européenne a très tôt engagé un combat qui fédère en réalité toutes les femmes ostracisées d'une manière ou d'une autre, en Afrique par contre, de nombreuses pesanteurs ont retardé cette échéance, la tenant à bonne distance du phénomène de la mondialisation. Comme le relève Aminata Sow Fall, le " passage du pilon à la machine à écrire " (1983, p. 73) signifie la venue très tardive de la femme à l'écriture. Elle ne s'est pas faite aussi facilement qu'elle ne l'a été pour l'homme. En effet, le premier roman féminin africain, Ngonda, de Marie-Claire Matip (1958), paraît trentesept ans après le premier roman africain masculin (1921). C'est que, en Afrique, la prégnance de la société phallocratique et son corollaire le confinement de la femme au rôle de mère au foyer n'ont pas favorisé l'émergence de son éveil au plan littéraire. C'est également cette venue tardive de la femme au-devant de la scène littéraire qui explique son réveil tardif à travers les diverses instances de plaidoyer mises sur pied en vue du respect de ses droits.

Pourtant, à bien y voir, une lecture attentive de Le journal intime d'une épouse d'Angéline Solange Bonono, d'Une vie hypothéquée d'Anne-Marie Adiaffi, et de Le briseur de rosée d'Edwidge Danticat, distantes de vingt-trois ans pour les deux premières et de trois ans pour les deux dernières, n'oblige-telle pas à établir une filiation sémantique plurielle et sous-jacente entre ces écrivaines et bien d'autres dans le cadre de la mondialisation? Par ailleurs, n'importe-t-il pas de positionner ces écrivaines comme militantes tant elles travaillent pour définir la place de l'écrivaine africaine dans le concert des nations du monde ? Bien plus, ne convient-il pas de s'interroger sur la nature des relations entre la Côte d'Ivoire (Afrique occidentale) et le Cameroun (Afrique centrale), Haïti (Amérique) et le monde féminin à travers le plaidoyer 
féminin pour l'avènement d'une nouvelle femme et l'éclosion d'un type nouveau de relations, au-delà des frontières linguistiques (langues africaines, français et créole), et géographiques (Afrique et Amérique)?

Le référentiel de lecture qui oriente notre réflexion se réclame du comparatisme et de l'approche genre. La littérature comparée se révèle « l'art méthodique, par la recherche de liens d'analogie, de parenté d'influencer, de rapprocher la littérature des autres domaines de l'expression ou de la connaissance, ou bien les faits et les textes entre eux, distants ou non dans le temps ou dans l'espace » (BRUNEL, PICHOIS, ROUSSEAU, 1983, p. 150). Elle permet d'afficher les passerelles qui existent entre des textes issus de cultures différentes. Voilà pourquoi Brunel et al. en conditionnent l'opérationnalité en soutenant: " pourvu qu'ils [les textes examinés] appartiennent à plusieurs langues, ou plusieurs cultures fissent-ils partie d'une même tradition afin de mieux les décrire, les comprendre et les goûter » (BRUNEL, PICHOIS, ROUSSEAU, 1983, p. 150). La variante genre convoquée renvoie à l'antinaturalisme féministe de Cynthia Kraus. Elle s'inaugure par un questionnement: « entre sexe et genre: où est le corps ? (2003, p. 39). Kraus entend ainsi réaffirmer l'omniprésence du corps dans le combat féministe en vue de l'implémentation de l'approche genre dans les rapports sociaux de sexe. Pour elle, le corps « ne disparaît pas à n'importe quel moment dans l'histoire de la pensée féministe » (KRAUS, 2003, p. 39). Car si la question de sa disparition ne se pose pas, c'est parce que les féministes s'engagent plus que jamais à " dénaturaliser la nature : tant et si bien, en effet, qu'elles sont sur le point de faire disparaître la nature, biologique en particulier, en la subsumant entièrement sous la culture ou le social " (KRAUS, 2003, p. 39). Autrement dit, l'idéal critique de l'antinaturalisme féministe, qui se confond dans son approche à l'épistémologie du genre dans son versant constructionniste, vise à " en finir avec la nature en réduisant totalement le biologique au socioculturel » (KRAUS, 2003, p. 39). C'est ce que Kraus qualifie d'« avarice épistémique $"$.

La présente étude s'organise autour de trois pôles opératoires. D'abord, on scrute la place de l'écrivaine africaine dans le concert des nations du monde. Ensuite, on interroge les relations qui la lient aux autres écrivaines sous la bannière de la mondialisation. Enfin, on montre que le message que 
DE LA TEXTURE DES DIÉGĖSES DES ÉCRIVAINES DE LA POSTCOLONIE À LA CONTEXTURE D'UNE MONDIALISATION ALTERNATIVE

relaie les femmes des aires linguistiques et géographiques différentes les positionne comme les chantres du vivre ensemble au moment même où certains pays du monde font face à des velléités sécessionnistes.

\section{De la place de l'écrivaine africaine dans le concert des nations}

Lorsqu'il entreprend de définir les contours de ce qu'il nomme « les enfants de la postcolonie », Abdoulaye Wabéri choisit de les caractériser comme une "génération transcontinentale » (1998, p. 11). Cette génération réclame sa place au cœur de la mondialisation et tient à partager avec elle son frisson existentiel. Pour Wabéri ces enfants sont les " premiers à user sans complexe du double passeport, à jouer sur deux, ou trois ou quatre tableaux, à se considérer comme africains et à vouloir en même temps dépasser cette appartenance. [...] Aujourd'hui, on se voudrait d'abord écrivain et accessoirement nègre " (1998, p. 11). La production romanesque des écrivaines africaines de l'Ouest comme du Centre transcende donc les clivages raciaux, linguistiques ou géographiques. Elle s'inscrit davantage dans une veine qualifiante qui voit dans cette catégorie d'écrivains « une génération [nouvelle] par-delà les frontières " (WABERI, 1998, p. 11), lorsqu'on la confronte aux romancières de la littérature haïtienne. Autant affirmer que toutes les productions des écrivaines sus-évoquées contribuent à souligner leur vraie place dans le concert des nations. Elles y revêtent une double étiquette : celle de chantres de l'équité et d'apôtres de la nouvelle mondialisation.

\section{Les chantres de l'équité}

Henri Mitterand dans sa tentative de définir le roman, affirme : « tout roman propose à son lecteur, d'un même mouvement, le plaisir du récit de fiction, et, tantôt de manière explicite, tantôt de manière implicite, un discours sur le monde » (1980, p. 5). Cette définition illustre à quel point la littérature en général et le romancier en particulier, travaillent chacun dans le sens de briser les frontières qui s'érigent très souvent entre les peuples au point d'hypothéquer la mise en pratique de la mondialisation. Au regard de la thématique de l'affirmation identitaire qui se fait obsédante dans les productions littéraires de la gent féminine de par le monde et que sécrètent 
artistiquement le roman féministe africain et haïtien, force est de dire que le "discours sur le monde » dont il s'agit ne confine plus l'écrivaine dans les carcans d'une géographie spécifique. Ledit discours se veut transcendantal en ceci qu'il œuvre au dépassement des frontières afin de remettre au goût du jour l'universalité d'un malaise féminin expérimenté dans la plupart les territoires anciennement colonisés. Le roman devient alors « un miroir qui se promène sur une grande route " (STENDHAL, 1989, p. XXVIII), la route du monde entier. Le discours auquel ont recours les écrivaines permet, de ce point de vue, de visualiser la dimension « mondialisante » des problématiques auxquelles sont soumises les femmes dans la mesure où il fédère l'art littéraire interafricain (Cameroun et Côte d'Ivoire), et celui des Amériques, notamment d'Haïti. La place qu'occupe l'écrivaine dans l'espace mondial des Lettres devient celui de guide, de porte-étendard.

Le guide montre la voie et donne de la voix pour canaliser la troupe afin de la préserver d'un danger qui se veut permanent. Si Wabéri en arrive à taxer les écrivaines africaines de " génération par-delà les frontières ", c'est parce qu'elles se déploient dans la perspective de créer des passerelles et de susciter des espaces d'échanges féconds en vue de réfléchir ensemble à l'émergence d'une cité africaine et américaine éthique sous-tendue par l'approche genre et la modernité. Voilà pourquoi un critique soutient que " quand une femme africaine se met à écrire, elle est [mue] par l'envie de changer les valeurs établies qui embarrassent son émancipation. Son écriture est signifiée par le désir de décrire sa propre souffrance. C'est une écriture d'amertume, une plaidoirie "féministe" " (GRESENGUET, 2013, p. 47). L'écrivaine africaine relativise et internationalise en même temps son combat en soulevant les problématiques auxquelles est confronté son peuple aux fins d'en procéder à l'exorcisation, en sollicitant peu ou prou le concours d'autres féministes.

Dans leurs diégèses respectives, Adiaffi et Bonono soulèvent la question de la réification de la femme et de l'enfant, deux catégories du genre. Tout comme le fait Danticat au regard des principes comportementaux édictés par les Objectifs du Millénaire pour le Développement (OMD). Dans le numéro 2 desdits objectifs par exemple, il est demandé d'« assurer une éducation primaire pour tous ", tandis que le numéro 3 recommande de "promouvoir 
DE LA TEXTURE DES DIÉGĖSES DES ÉCRIVAINES DE LA POSTCOLONIE À LA CONTEXTURE D'UNE MONDIALISATION ALTERNATIVE

l'égalité des sexes et l'autonomisation des femmes ». Chez Adiaffi, la jeune fille tombe sous le coup des considérations essentialistes des rapports sociaux de sexe. Sa nature de femme l'emporte sur sa culture, tant l'homme la considère comme un objet et non un sujet dans sa propre histoire. Sa capacité à s'affirmer comme une créature digne de respect et capable de décider par elle-même lui est contestée. Chosifiée, réifiée, son avenir est hypothéqué puisqu'elle est troquée sans son avis et comme un objet de change contre de meilleures conditions de vie pour ses parents pauvres. Regardée comme un sexe « bénéfiquement négociable » (1984, p. 7), Ya est trop tôt mariée à au richissime Béhira, un homme d'affaire présenté par le narrateur comme un "solide gaillard d'une cinquantaine d'années [...qui] trainait dans sa culotte l'une des plus grosse "carottes" dont un mâle pouvait disposer " (ADIAFFI, 1984, p. 9-10). Cette occurrence souligne la gravité des faits car, non seulement Béhira fait office de pédophile, mais aussi il est viole les règles du droit de la femme à disposer de sa vie à l'âge nubile.

Ce manquement à l'éthique relevé dans une Côte d'Ivoire stylisée devient une problématique universelle qui court les cités du monde tant elle se trouve régulièrement relayée aussi bien par la presse que les média du monde. L'œuvre de la camerounaise Angéline Solange Bonono s'en fait l'écho lorsqu'on y voit Esther, jeune bachelière de dix-neuf ans, vendue à Crésus, un riche homme d'affaire. Mû comme Béhira par la veine essentialiste dans le regard qu'il pose sur la femme, ce dernier pousse loin le vice du mépris en ne prenant même pas part à la dot de sa future épouse. La narratrice omnisciente affirme : " j'ai été dotée la veille par procuration. Des noces arrangées [...]. Ma famille est heureuse que j'épouse un milliardaire car elle espère que je vais changer sa condition" (BONONO, 2007, p. 8). La thématique de la chosification de la femme traverse également la diégèse d'Ewige Danticat, entraînant ainsi une relation implicite entre les écrivaines d'Afrique et celles de l'Amérique sous le label de la mondialisation, à travers des échanges littéraires.

Un clin d'œil rétrospectif sur la grande Histoire d'Haïti permet effectivement d'établir un lien étroit entre l'accession à la magistrature suprême de François Duvalier, en 1957, et l'occurrence textuelle " vers 1967 » (DANTICAT, 2004, p. 227) et la réification de la femme par le personnage éponyme. Cet indice historique confirme la volonté affichée de la romancière 
de replonger le lecteur dans les dédales d'un contexte historique caractérisé par affres du duvaliérisme ${ }^{2}$. Dans le récit, l'indice historique relatif aux miliciens dudit système permet de relever la prégnance des maux tels que l'abus d'autorité et la crise des droits de l'Homme. La place de guide qui spécifie la mission de l'écrivaine africaine et haïtienne en fait également le garant de la subversion de toute forme de stigmatisation et d'exploitation notée à l'endroit des enfants et des femmes. Au moyen de la littérature et par-delà ses interviews et autres échanges accordées ci et là dans les médias et les fora, l'écrivaine entonne l'hymne de la revendication en vue d'affranchir l'humain des chaînes de la barbarie. En cela, le combat des femmes africaines et celui de l'américaine se rejoignent. Tirant avantage d'un système politique qui ne profite qu'à ses partisans les plus fidèles, à la manière des phallocrates qui ne pensent qu'à satisfaire leurs intérêts, les miliciens s'illustrent comme les bénis de la dictature duvaliériste. Sans respect pour les droits humains, ils agissent sans foi ni loi. L'abus d'autorité dont ils font montre oblige à comparer le système dictatorial haïtien aux pratiques androcentriques en vigueur dans les récits africains. Favorable aux hommes comme il l'est pour les "papas docs", il s'étend à plusieurs niveaux de la vie sociale. Le narrateur nous en donne la preuve à travers cette assertion : « Des bourgeoises mariées dormaient avec lui [la nouvelle recrue] sur le matelas rempli de billets de banque [...] des vierges issues de toutes classes sociales entraient et repartaient également » (DANTICAT, 2004, p. 244). A l'image des phallocrates obligent la femme à se dresser contre sa consœur, ainsi les miliciens contraignent-ils les citoyens au musellement à l'instar du pasteur qui est assassiné ao cœur de sa case chapelle. Pour libérer la femme et le peuple du giron phallocratique, de la servitude et de l'escalade de l'ostracisation qui l'enserrent, l'écrivaine revêt la camisole du porte-étendard.

A la manière dont un athlète porte haut l'étendard d'un peuple et s'assure à la tête de la file d'échanger avec les autres nations de la terre en défendant les couleurs qu'il arbore à l'ouverture des Jeux Olympiques, telle est

\footnotetext{
${ }^{2}$ Le duvaliérisme désigne le régime mis en place en Haïti par le dictateur François Duvalier dit « Papa Doc », arrivé au pouvoir après un putsch en 1957, et poursuivi par son fils Jean-Claude Duvalier, dit «Baby Doc » entre 1971 et 1986. Ce régime s'appuie sur une partie de l'armée et une milice paramilitaire, les Tontons Macoute. https://fr.wikipedia.org/wiki/Duvali\%C3\%A9risme
} 
DE LA TEXTURE DES DIÉGĖSES DES ÉCRIVAINES DE LA POSTCOLONIE À LA CONTEXTURE D'UNE MONDIALISATION ALTERNATIVE

le statut qui incombe au romancier dans la dynamique de postulation d'une mondialisation des cultures entre écrivaines d'Afrique et celles de l'Occident. Robert Escarpit traduit cette mission en rappelant l'horizon d'attente que manifeste le livre pour le peuple. Pour lui, « le livre à succès est le livre qui exprime ce que le groupe attendait, qui révèle le groupe à lui-même. [...] On peut donc dire que l'ampleur du succès d'un écrivain à l'intérieur de son groupe est fonction de son aptitude à être l'écho sonore dont parle Victor Hugo » (ESCARPIT, 2007, p. 110). Aucune nation digne de ce nom ne souhaite vivre en autarcie. En d'autres termes, les attentes du peuple telles que formulées par les écrivaines se voient davantage dans le rapport qu'entretiennent le social et le littéraire, puisque «c'est le lecteur qui est le "producteur du texte", l'écrivain est l'homme de peine qui pousse des brouettes de mots pour la construction d'un édifice dont seul le lecteur "scriptible" averti connaît le grandiose dessein " (GORDIMER, 1998, p. 29). Ainsi, si la formation sociale produit sa littérature, en retour, « celle-ci produit du social selon des effets dont on ne mesure pas toujours l'importance » (DUBOIS, 1987, p. 290). Plusieurs indices confortent cette assertion dont les ressorts sont l'intertextualité, l'intermédialité, les phénomènes de la citation et de l'allusion et l'usage des culturèmes.

L'intertextualité renvoie à l'idée que « nul texte ne peut s'écrire indépendamment de ce qui a déjà été écrit et il porte, de manière plus ou moins visible, la trace d'un héritage et de la tradition » (PIEGAY-GROS, 1996, p. 7). A travers ce phénomène transculturel, les écrivaines africaines et américaines se rapprochent, établissant des ponts entre leurs diverses cultures au nom d'une mondialisation qui s'impose à eux grâce à l'interaction qu'elle impulse tacitement. Les romancières sus-évoquées manifestent l'usage significatif de cette stratégie d'écriture qui les place les unes au confluent des autres. Dans Le briseur de rosée, c'est "le livre des morts» allusivement évoqué par le personnage éponyme, le briseur de rosée, qui permet aux écrivaines d'Amérique et d'Afrique de nouer une relation significative qui dépasse le simple cadre de la géographie. Sujet essentiellement violent et instrumentalisé sous les Duvalier, ce personnage entend se bâtir une nouvelle identité fondée sur l'oubli des erreurs du passé. Il lui faut confesser ses fautes afin de renaître à la vie. Il verse alors dans des aveux pathétiques aux fins de convoiter le 
pardon de sa fille Ka, à qui il a caché son passé, en se culpabilisant à ses yeux : « Ka, ton père était le chasseur, il n'était pas la proie [...]. Je travaillais en prison [...] c'est l'un des prisonniers de la prison qui m'a fendu le visage ainsi [...] L'homme qui m'a tailladé la joue [...] je l'ai abattu comme j'ai tué beaucoup de gens » (DANTICAT, 2004 : 32). Cette espèce de confession négative inspirée d'un des cérémonials du Livre des morts des Anciens Egyptiens datant de 1842, absout les péchés du briseur de rosée et l'affiche comme un homme nouveau, corps, âme et esprit, celui qui peut désormais « sortir au jour » et redémarrer le train de sa vie, en se positionnant comme le chef d'une famille unie et forte, fondée autour d'une synergie, celle de ses membres. Voilà pourquoi dans la littérature égyptologique moderne, la juxtaposition des deux titres, à savoir "Livre des Morts - Sortir au jour » se rencontre souvent. De même, dans Une vie hypothéquée, l'allusion à la Deuxième Guerre mondiale permet au lecteur de tisser un lien implicite entre le roman ivoirien et l'Histoire de l'Allemagne voire du monde entier par la voix de l'écrivaine Adiaffi. Bien que relatant les affres de la fille réifiée, son texte ouvre une brèche pour se révéler un espace de diffusion de la culture mondiale dans ce qu'elle a connu en termes de souvenirs atroces. Le vieux Béhira évoque les indices renvoyant à la Deuxième Guerre mondiale à travers de nombreuses allusions évasives à Baden Baden, aux tirailleurs sénégalais ainsi qu'à la personnalité d'Hitler dont il dresse ainsi le portrait comique: «Au cours du combat [...], Hitler se rendait invisible pour faire sauter la tête de tous les soldats ennemis. Les quelques rares individus qui avaient eu le privilège de le voir en chair et en os, disaient que sa taille était nettement supérieure à trois mètres » (ADIAFFI, 1984, p. 44).

Des trois romans en examen, seul Le journal intime d'une épouse fournit un riche éventail de liens qui inscrivent cette œuvre dans la mondialisation. L'évocation du poète Prévert autant que celle de Rousseau et de Mariama Bâ (BONONO, 2007, p. 48-58-62), montrent que le roman africain ne git pas dans la marginalisation mais que les écrivaines actionnent les leviers pour s'ouvrir aux autres littératures environnantes, surtout lorsque la thématique abordée paraît la même. Tel est le cas pour le roman de Bonono et Une si longue lettre de Mariama Bâ qui tous les deux se penchent sur la condition de la femme. Le narrateur Esther note au sujet du recoupement des deux diégèses: «c'est fou ce que peuvent vivre les femmes! Ce roman a été 
DE LA TEXTURE DES DIÉGĖSES DES ÉCRIVAINES DE LA POSTCOLONIE À LA CONTEXTURE D'UNE MONDIALISATION ALTERNATIVE

publié en 1979 ! Ça n’a pas beaucoup changé. J'ai été saisie par la similitude des situations entre Binetou et moi " (BONONO, 2007, p. 62). Le style adopté par Bonono dans son récit ainsi que la similitude des problématiques y traitées, obligent à penser à une situation de réécriture, c'est-à-dire une relation de production littéraire profonde entre les écrivaines camerounaise et sénégalaise. Par ailleurs, l'usage par Bonono des citations inspirées de Stassart, Rousseau, ou encore d'Aristote, de Zundel ou d'Hugo, affechent l'écrivaine camerounaise comme interface entre la littérature africaine et les littératures du monde. Si chaque citation évoquée correspond à un moment clé de la vie de l'héroïne Esther dans le récit, elle installe davantage la romancière camerounaise dans un lien dynamique fécond avec les autres cultures sous la bannière de la mondialisation. Convoquant par exemple Stassart, Bonono entend montrer à quel point le mariage polygamique constitue une enfreinte de la liberté de la femme : « quand on n'a plus sa liberté, il importe fort peu si la cage est dorée » (BONONO, 2007, p. 26). L'évocation des rapports qu'entretiennent les écrivaines africaines et américaine avec les autres littératures du monde consacre leur inscription au cœur de la mondialisation.

\section{L'écrivaine, un apôtre de la mondialisation}

La finalité espérée de la mondialisation était d'impulser une dynamique de partage, de créer une franche passerelle d'échanges fructueux entre territoires du monde à travers des modules culturo-identitaires précis permettant à chacun, partout où il se trouve, d'afficher son identité en vue de transformer le monde en un authentique village planétaire, un paradis terrestre, au sein duquel il fait bon vivre parce que tous les hommes s'y sentent frères. Le tourisme aurait pu être cette plateforme-là, ce lieu chargé d'espoir pour tous, entendu qu'il offrait aux âmes venues d'horizons divers de converger vers un seul but: échanger, apprendre les uns des autres. Il aurait pu, comme l'affirme Isabelle Mette, se révéler une "situation d'échange idéale " (2005, p. 32). Pour Michel Houellebecq, « ce que l'on appelle mondialisation, qui est l'uniformisation par le bas, le règne des multinationales, la standardisation, l'ultralibéralisme sauvage sur les marchés mondiaux, pour moi c'est le revers négatif d'une réalité prodigieuse, que j'appelle la mondialité » (2001, p. 152). 
Bref la mondialisation apparaissait comme le « contrepoids à une vision unilatérale du monde et pour faire voler en éclats certaines certitudes acquises » (METTE, 2005, p. 32). C'est à ce niveau de déchéance de son piédestal unilatéral en réalité que les écrivaines africaines et américaine, revêtues de leurs atours féministes tels que le suggère Leclerc, travaillent dans le sens de réinventer la notion même d'échange. Il s'agit de montrer par exemple que « le tourisme, loin d'être peint sous les couleurs tant vantées de la rencontre des cultures, [...] apparaît comme un lieu de tensions extrêmes " (METTE, 2005, p. 32). Les écrivaines inscrivent leur présence dans la mondialisation à travers un certain nombre de stratégies: l'affirmation de la culture africaine à travers l'usage des culturèmes et l'intermadialité.

Jean-Marie Privat considère que le culturème participe de la description et de l'affirmation identitaires d'un individu. Nul ne va de ce point de vue à la rencontre de l'autre en oubliant chez lui ses attributs identitaires. C'est cette nouvelle forme de mondialisation que postulent les écrivaines, celle qui procède de la « réinvention de la notion d'échange » (METTE, 2005, p. 34). La mondialité, nouveau paradigme définitoire de la mondialisation actuelle, s'exhibe dès lors comme « l'aventure sans précédent qu'il nous est donné à tous aujourd'hui de vivre, dans un monde qui pour la première fois, réellement et de manière immédiate, foudroyante, se conçoit à la fois multiple et unique, et inextricable. C'est aussi la nécessité pour chacun d'avoir à changer ses manières de concevoir, de vivre et de réagir dans ce monde-là " (HOUELLEBECQ, 2001, p. 152). A l'instar de la mission assumée par les apôtres du Christ, celle des écrivaines se précise. Il s'agit d'afficher les attributs culturels de leur ressort géographique afin de les poser sans les opposer à ceux des autres, mais simplement dans le but de promouvoir son identité.

Dans Le briseur de rosée comme dans Une vie hypothéquée, Adiaffi et Danticat font usage de l'interlangue en utilisant des mots en langues nationales africaines dans un texte en langue française. Le souci ici est d'afficher son appartenance culturelle tout en travaillant à la diffusion de sa culture. Le principe de mondialité devient opérant à partir du moment où l'écrivaine donne une traduction du mot ou de l'expression utilisée afin de ne pas s'enfermer dans les carcans de l'auto-marginalisation. Dans Une vie hypothéquée, on peut lire: « ottè kokorè ! Ottè kokorè kê wo ! (Il est clair Une 
DE LA TEXTURE DES DIÉGĖSES DES ÉCRIVAINES DE LA POSTCOLONIE À LA CONTEXTURE D'UNE MONDIALISATION ALTERNATIVE

vie hypothéquée, il est clair comme toi ! (ADIAFFI, 1984, p. 30). Ou encore: " ottè-sè ? (Comment est-il ?) » (ADIAFFI, 1984, p. 31). L'écrivaine prend le soin de traduire entre parenthèses l'expression utilisée afin de faire passer le message. Tel est également le cas dans Le briseur de rosée lorsque le père parle ainsi à sa fille $\mathrm{Ka}$ : « je t'ai appelée $\mathrm{Ka}$ [...] Tu vois, Ka est comme l'âme [...]. En Haïti, c'est ce qu'on appelle un bon ange, ti bon anj " (DANTICAT, 2004: 27). Dans cette occurrence, le père de Ka met en exergue la signification culturelle profonde du nom qu'il lui a attribué après l'avoir cerné comme son ange.

L'usage des culturèmes est aussi relevé à travers l'inscription des proverbes dans le récit des écrivaines. Il s'agit pour elles d'exposer à la face du monde la stratégie de l'art oratoire dans les anciennes colonies. Ici un argument force tire sa puissance de l'émission d'un énoncé sentencieux qui résume le sens du propos tenu. Visant à justifier l'acte de vendre sa fille pour résorber son problème de pauvreté, Kouamé le père de Ya affirme: « nul ne refuse de croquer le morceau de sucre qui lui tombe dans la bouche " (ADIAFFI, 1984, p. 6). Dans le même ordre d'idées, alors que la narratrice omnisciente et épouse de polygame s'est momentanément retirée chez ses parents se mettant ainsi à l'abri des viols dont elles sont les victimes par la faute des chauffeurs de son époux, elle préfère ce mode de conduite à celui consistant à affronter les violeurs en reconnaissant dans Le journal intime d'une épouse: « mieux vaut dix lâches vivants qu'un lion mort »(BONONO, 2007, p. 96). Parfois, l'un des traits identitaires des écrivaines africaines réside dans le choix de contextualiser certains usages linguistiques en les parodiant, comme pour leur insuffler une touche locale. Dans Le journal intime d'une épouse, la narratrice camerounaise communique avec un auteur ivoirien, Birago Diop, à travers la parodie " les morts sont morts » (BONONO, 2007, p. 93) pour dire le contraire, soit «les morts ne sont pas morts». De même, pour jurer d'être en train d'adapter l'usage au mois au cours duquel l'expression connue "poisson d'avril " est prononcée, la narratrice dira plutôt: "Sophie a été libérée et ce n'est pas un poisson d'août" (BONONO, 2007, p. 90). Au regard de ce qui précède, il y a lieu d'avaliser l'argument que «[1]e monde est donc celui de la mise en contact, de l'échange, celui de la prise de conscience qu'il existe une pluralité de façons de concevoir le monde. C'est cette diversité que la littérature peut par ailleurs donner à voir » (METTE, 2005, p. 34). L'inscription 
de l'intermédialité participe également de cette dynamique de réinvention de la mondialisation à travers le texte féminin.

L'intermédialité permet d'illustrer le cosmopolitisme qui traverse parfois le roman africain à travers le contact qui se noue entre lui et d'autres médias. L'intermédialité dévoile l'autre facette de la mondialisation à savoir, l'esthétique de l'hybridité, ou du mélange intermédias qui inonde le récit et autorise l'écrivaine d'origine africaine ou antillaise à proclamer, sur le mode de la paraphrase, sa statut hybride car les écrivaines font « l'Eloge de la créolité ». Ainsi partagées entre afropolitanisme et afropéanisme grâce en partie à l'usage de l'intermédialité, lesdites écrivaines cessent d'être en réalité antillaise (Danticat) ou encore africaines (Adiaffi et Bonono), et deviennent tout simplement créoles (BERNABE et al., 1989, p. 13). Autant admettre que l'usage de l'intermédialité positionne les écrivaines du continent noir et de celles de la diaspora comme des citoyennes du monde, parce que façonnées dans un moule cosmopolitique. Dans Le journal intime d'une épouse, grâce à l'art cinématographique manifesté à travers l'usage d'internet par deux des épouses martyrisées dans le harem de Crésus, les femmes passent de la marginalité à l'altérité pour ne pas dire à la mondialité. Car, enfermées dans le harem d'un époux invisible, elles ne doivent leur salut qu'à la correspondance avec des amants internet qui leur ouvrent la voie du bonheur jusque-là hypothéqué chez leur époux. La narratrice confie au lecteur: "nous avons trouvé des correspondants sur internet, des amants pour l'instant platoniques. Le mien est camerounais et vit à Enghein-les-bains, celui de mon amie est français et réside à Paris » (BONONO, 2007, p. 27). Euvrant dans la perspective de se sortir du carcan polygamique, de celui de l'essentialisme (marginalité), à celui du constructionnisme (leur projection hors des frontières de l'Afrique pour la liberté conjugale), les deux épouses recourent à une arme à laquelle personne n'avait songé en réalité : internet, l'art filmique, puisqu'on voit celui à qui on parle et vice versa. Le mariage à nouveaux frais de Félicité et d'un Blanc qui naîtra de cette union lointaine par le biais d'internet instaure un autre mode de déclinaison de la mondialisation entre deux continents à travers les écrivaines en examen. Ainsi, le caractère polymorphe qui est caractéristique de l'intermédialité établit une passerelle entre des mondes éloignés en apparence 
DE LA TEXTURE DES DIÉGĖSES DES ÉCRIVAINES DE LA POSTCOLONIE À LA CONTEXTURE D'UNE MONDIALISATION ALTERNATIVE

mais proches en réalité à travers les écrivaines africaines et antillaise dont les personnages relaient la vision du monde.

\section{La vision du monde des écrivaines}

Dans l'épistémologie sociocritique d'Henri Mitterand, le texte de roman « par le travail de l'écriture, [... articule] un discours sur le monde » (1980, p. 7). L'écriture, de ce point de vue modifie l'équilibre antérieure du sens et définit la vision du monde qui anime le romancier. Elle se décline à travers deux modalités : le déni de la marginalisation et la célébration de l'altérité.

\section{Le déni de la marginalisation}

Restreindre notre approche au décryptage de la place des écrivaines et les enjeux de la mondialisation au simple plan scripturaire relèverait en soi de la marginalité, puisqu'on sait que le discours essentialiste se veut fondamentalement marginal : il confine la femme au rang de "deuxième sexe » (BEAUVOIR, 1949, 11). Le déni de la marginalisation procède donc d'une nouvelle forme de rébellion féminine, anti-essentialiste, qui émane du désir affiché par les écrivaines d'ouvrir leurs œuvres au monde et s'ouvrir elles-mêmes à travers des rencontres diverses au centre desquelles figurent des échanges divers. On peut évoquer le cas du Dictionnaire littéraire des femmes de langue française de Christiane P. Makward et Madeleine Cottenet-Hage, toutes deux écrivaines et enseignantes aux Etats-Unis. L'idée d'un rassemblement transnational fédérant certaines écrivaines dans le cadre d'un ouvrage bannit un tant soit peu les marges tout en conjurant les exclusions. L'ouvrage en question regroupe 200 notices rédigées par des critiques françaises et nord-américaines sur des écrivaines de langue française de toutes les aires de la francophonie, des origines (XIIe siècle) à nos jours. Le déni de marginalisation naît aussi du désir de rendre justice à des écrivaines méconnues mais qui travaillent dans l'ombre pour inscrire leur travail au cœur de la mondialisation. Ceci explique pourquoi ce dictionnaire comporte, en outre, environ 900 bibliographies d'auteurs n'ayant pu faire l'objet d'une analyse ou ayant trouvé jusqu'à présent qu'une 
place marginale dans les ouvrages de référence. La production littéraire s'avère ainsi un espace de compensation des manquements relevés au plan national.

On peut en outre mentionner Le féminin des écrivaines Suds et périphérie co-dirigé par deux écrivaines d'origines géographiques diverses à savoir, Françoise Moulin Civil de France et Christiane Chaulet Achour d'Algérie. Cet essai critique mobilise diverses sensibilités d'écrivaines issues d'horizons géographiques et linguistiques multiples. Il célèbre en réalité le caractère fédérateur permettant de redonner vie aux écrivaines du Sud et de sa Périphérie. Il s'agit dans cet espace d'expression féministe de combattre les formes de rejet qui exacerbent les essentialismes et accentue les marges. La militance interpelle ainsi toutes les sensibilités " écrivantes ». Le refus de la marginalisation se voit lorsque les écrivaines embrassent en masse l'idée du voyage et suscitent des espaces d'échanges fructueux pour regarder en face la problématique du monde telle que soumise aux aléas de son devenir conciliateur. Wabéri conforte cette allégation en affirmant : " ces écrivains [de la postcolonie] voyagent beaucoup - mais de plus en plus difficilement depuis que les frontières de Schengen se sont refermées comme des huîtres " (WABERI,1998, p.14). Les Camerounaise Léonora Miano, Angéline S. Bonono et tout comme Nathalie Etoke vivent ou ont vécu en Occident (France, EtatsUnis) où elles travaillent au confluent d'autres écrivaines qui inspirent leur écriture et alimentent indirectement leur combat à l'instar de Calixthe Beyala. Cette dernière donne le témoignage de cet échange sur fond de combat féministe dans un essai, Lettre d'une Africaine à ses sœurs occidentales. Son style franc et engagé s'en ressent chez une Etoke ou une Bonono lorsque cettedernière fait dire à son personnage en recourant à une interrogation rhétorique : " la femme est-elle un animal nuisible pour être harcelée, serrée de près, rabattue comme du gibier vers les lignes de tir, poursuivie sans relâche par la merde ? " (BONONO, 2007, p. 37). La France, espace ouvert au nouveau sens de la vie par le biais des échanges militants devient le terreau fécond de la mondialisation lorsqu'on sait, par ailleurs, que Danticat, de souche haïtienne, évolue elle-aussi aux Etats-Unis, notamment à Brooklyn qu'elle nomme dans son récit. Cet argument se trouve conforté lorsqu'on sait que « beaucoup de ces écrivains[nes] se sont rencontrés et ont échangé des idées et des points de vue à l'occasion des forums et des festivals, comme celui de Limoges où la plupart 
DE LA TEXTURE DES DIÉGĖSES DES ÉCRIVAINES DE LA POSTCOLONIE À LA CONTEXTURE D'UNE MONDIALISATION ALTERNATIVE

d'entre eux ont été soit résidents soit visiteurs de passage " (WABERI, 1998, p.14). Il va sans dire que les rencontres démultipliées entre écrivaines dans le cadre d'échanges fertiles en Occident appellent l'invention d'une nouvelle mondialisation qui célèbre véritablement l'altérité.

\section{La célébration de l'altérité}

La célébration de l'altérité rejoint par contre les axes opératoires caractéristiques de l'approche constructionniste, tant elle interdit d'en rester à la nature pour postuler la mondialité. Elle pose la problématique de l'invention d'un nouvel Homme mondial dont le visage est dessiné en filigrane dans les récits des écrivaines. Il s'agit pour ces dernières d'asserter que " toute œuvre littéraire a ses traits individuels ; mais elle partage aussi certaines propriétés avec d'autres œuvres " (WELLECK, WARREN, 1971, p. 22). A cet égard, si les culturèmes sont mis en scène dans les romans africains et ceux de la diaspora, c'est davantage dans l'optique de pérenniser une personnalité et d'affirmer une identité sans laquelle l'altérité n'existerait pas. Car, c'est à l'autre que je montre qui je suis au fond afin d'échanger avec lui ce que j'ai tout comme ce qu'il possède. Le gain par Nadine Gordimer, écrivaine sud-africaine, du Prix Nobel de Littérature, tout comme celui du Grand Prix Littéraire d'Afrique Noire par Calixthe Beyala, écrivaine camerounaise, montrent par exemple que l'altérité de demain passe par la reconnaissance aujourd'hui des performances cognitives de l'écrivaine d'aujourd'hui. Tel est aussi le sens profond qu'il convient d'attribuer à la veine intertextuelle et intermédiale qui sédimente les romans sus-évoqués. Car, en convoquant Stassart, Prévert, mais surtout une autre femme, Mariama Bâ dans son roman, Bonono dévoile implicitement ses sources, la nature des échanges tacites qu'elle e eue avec ses modèles femmes. De même, en fondant en 1987 le Centre Africain d'Animation et d'Echanges Culturels (CAEC), en publiant des romans qui sont autant lus en Afrique qu'aux Etats-Unis, en voyant ses œuvres traduites en plusieurs langues, et en se faisant élever au grade de Docteur Honoris Causa dans de nombreuses universités étrangères, Aminata Sow Fall, écrivaine sénégalaise, proclame l'urgence de susciter de nouveaux modules d'échanges en vue de la rédaction de nouvelles pages d'une mondialisation alternative. Cette dernière doit 
devenir une formidable ligne de partage, un champ de savoirs altruistes et non un ring où les écrivaines de lancent de défis inopérants. Voilà pourquoi Thierno Monénembo voit dans l'exil des écrivains une instance futuriste en vue du devenir du monde en affirmant qu'il faut " profiter de l'exil » (dans WABERI, 1998, p. 15).

Wabéri quant à lui inscrit ses espérances en droite ligne de celles placées en une mondialisation neuve consistant à générer autour des femmes écrivaines du monde une synergie d'actions concertées dont le point culminera est l'éclosion d'une Terre faite de concessions et de compromissions. Dans une verve proleptique empreinte de réalisme, il confie ceci au sujet des enfants de la postcolonie : « au final, cette quatrième génération apportera quelque chose de neuf et justifiera son existence; si elle transforme l'exil d'ordinaire angoissant, annihilant, douloureux, en un exil fécondant, joyeux, qui n'est plus appréhendé sur le mode nostalgique, souffreteux, en un mot, "doloriste" » (1998, p. 15). Mais plus encore, la génération des enfants de la postcolonie à laquelle appartiennent entre autres Fatou Diome, Sénégalaise vivant à Strasbourg en France, et Nathalie Etoke, Camerounaise vivant aux Etats-Unis, milite à distance dans des forums tels que le Congrès International des Recherches Féministes dans la Francophonie (CIRFF). L'intention derrière cet espace de mobilisation des chercheurs et écrivaines est de sortir les unes et les autres de l'enfermement géographiques afin de "Penser, Créer, Agir » ensemble suivant les termes de leur slogan. L'écrivaine sert ici d'interface dans le combat entre la société logocentrique et le monde nouveau dessiné dans l'écriture pour le respect des droits des femmes en vue de la postulation d'une mondialisation alternative. Parodiant le communiste Karl Marx en 2018 à Nanterre en France, les chercheures travaillent aux côtés des écrivaines sous la bannière du postulat "Féministes du monde francophone, unissez-vous ! 1500 chercheuses et militantes au $8^{\text {ème }}$ CIRFF à Nanterre, du 27 au 31 août 2018. Dans son article y relatif, Nadia Bouchenni (2018) souligne l'enjeu fédérateur qui impulse des trajectoires neuves à une mondialisation qui se veut désormais alternative. Elle précise : "Initialement créé pour un public essentiellement universitaire et associatif, ce congrès reste assez scientifique dans sa démarche, mais est ouvert au grand public. Étudiantes, chercheuses, journalistes, mais aussi militantes, activistes et artistes de tous âges, de tous 
DE LA TEXTURE DES DIÉGĖSES DES ÉCRIVAINES DE LA POSTCOLONIE À LA CONTEXTURE D'UNE MONDIALISATION ALTERNATIVE

horizons et d'origines diverses sont présentes pour échanger, s'informer, et apprendre ${ }^{3} »$.

La nouvelle mondialisation ainsi conceptualisée ne se définit plus comme une asymptote de la "détérioration des "termes de la chance" [qui accorde la part belle à] l'échange inégal en littérature » (METTE, 2005, p. 28). Elle s'exhibe par contre comme le forum par excellence de la transcendance des frontières linguistiques et géographiques en vue de son positionnement comme espace des synergies opérantes et du rassemblement fécond. Il convient à cet égard d'affirmer que ce sont les Québécoises (Amérique) et les Sénégalaises (Afrique) qui sont à l'origine des premières éditions du CIRFF. Ses membres sont passés de 100 à 1500 inscrites. De plus, l'alternance du site des rencontres traduit l'impératif d'inscrire ladite mondialisation dans une dynamique de délocalisation et de rotation pour davantage en révéler le fondement de l'équité qui la sous-tend. Ses ressorts et assises égalitaires sont désormais la volonté affirmée d'impliquer toutes les parties prenantes à l'édification d'un monde neuf à l'instigation des écrivaines. Co-auteure avec Brigitte Rollet de Genre et légitimité culturelle. Quelle reconnaissance pour les femmes ?, Delphine Naudier conforte cette allégation dans l'article de Bouchenni. Elle affirme que la toute première édition du congrès a eu lieu en 1996 à l'Université Laval à Québec après une rencontre en 1995 au Brésil qui réunissait des féministes francophones. Le deuxième colloque eut lieu en 1999 à Dakar, avec l'idée de continuer à organiser le congrès dans des pays francophones, mais en changeant de continent à chaque fois. Après Québec (Amérique) et Dakar (Afrique), le congrès vient en Europe, à Toulouse plus particulièrement, en 2002. Puis Ottawa, Rabat, Lausanne, et à nouveau Québec, en 2015. La 8ème édition a donc choisi Paris, ou plutôt la banlieue parisienne, et pas en Afrique. La nouvelle mondialisation postulée suit son bonhomme de chemin puisque le CIRFF en est rendu à sa $8^{\text {ème }}$ édition.

Pour la sociologue Malika Hamidi, participante au $8^{\text {ème }}$ congrès et spécialiste du féminisme musulman, la nouvelle mondialisation scrutée à l'aune des rencontres internationales du CIRFF gagnerait à contribuer à la

\footnotetext{
${ }^{3}$ « Féministes du monde francophone, unissez-vous! 1500 chercheuses et militantes au $8^{\text {ème }}$ CIRFF à Nanterre » mis à jour le 30. 8. 2018, https://information.tv5monde.com/terriennes/feministes-du-mondefrancophone-unissez-vous-1500-chercheuses-et-militantes-au-8eme-cirff, consulté le 31 août 2019.
} 
décolonisation de la recherche dans le monde académique. La mondialisation devient ainsi " une source d'inspiration transcontinentale " pour reprendre Bouchenni. Elle s'ouvre non seulement à une variété d'espaces géolinguistiques, mais aussi à d'autres inspirations telles que celle du Parlement ${ }^{4}$ des écrivaines francophones. Près d'une soixantaine d'écrivaines provenant des cinq continents du monde ont participé à cette manifestation placée sous le patronage de Michaëlle Jean le 26 septembre 2018. Il s'agissait pour elles d'échanger sur les valeurs, les rêves et les combats communs rapprochant les cinq continents, de dialoguer en français leur véhicule d'écriture, puisque ledit Parlement ambitionne, d'après le site de la francophonie, rendre plus audible la voix des femmes, en leur offrant un espace de gestion de la prise de parole destiné à dévoiler le point de vue des écrivaines sur les débats ou les crises de nos sociétés, à encourager la présence féminine dans la prise des décisions et la résolution des conflits. Il s'agit donc d'un cadre d'échanges, de découvertes et de partage, une initiative de Fawzia Zouari, journaliste écrivaine tunisienne au bénéfice d'autres altérités. Ce projet est soutenu par Leïla Slimani, écrivaine franco-marocaine et chargée de mission francophonie auprès du Président de la République française, et sous l'égide de l'OIF (Organisation internationale de la Francophonie). Elle répond aux préconisations de l'Appel de Bucarest issu de la Conférence des Femmes de 2017. A l'instar du CIRFF, cet espace de rencontre a vocation, au fil des années, à voyager à travers le monde pour permettre à toutes les écrivaines francophones de se l'approprier. Autant croiser les doigts et alléguer à la suite d'Isabelle Mette que « si la littérature parvient ainsi à faire connaître et se rencontrer des voix jusqu'alors inconnues, on peut imaginer les premiers pas vers la “mondialité” » (2005, p. 35).

\section{Conclusion}

Au total, les écrivaines prennent fait et cause pour l'équité, non pour combattre l'homme, mais pour simplement donner une chance à l'histoire de rénover au travers de l'approche genre les rapports de sexe de par le monde. Ce rôle se veut capital dans l'éclosion de la littérature mondiale. Les relations

\footnotetext{
${ }^{4}$ Lire à ce sujet l'article de Victor De Sepausy du 18.03.2018 repéré dans https://www.actualitte.com/article/monde-edition/orleans-accueillera-le-parlement-des-ecrivainesfrancophones/87904. consulté le 30 août 2019.
} 
qu'entretiennent entre-elles les écrivaines sont à la fois endogènes, textuelles, à travers la fréquentation d'une ligne de partage thématique qui les rapproche implicitement, et exogènes, contextuelles, par le biais des rencontres physiques qui les mobilisent aux quatre coins de la planète au détour d'un Congrès ou d'un Parlement. Les voix féminines se définissent alors comme transcontinentales, tant elles claironnent par-delà des frontières linguistiques et géographiques qu'elles charrient, une forme nouvelle de mondialisation que certaines écrivaines appréhendent sous la bannière de la mondialité. Cette variante idéologique avalise le déploiement d'une vision constructionniste des rapports sociaux de sexes. Elle s'articule comme la modalisation des ressorts de la subversion de tous les égoïsmes essentialistes nés des pratiques phallocratiques qui renforcent l'ostracisation de la femme et instaure, au contraire, une vie nouvelle ancrée sur le vivre ensemble. Ses déclinaisons rejoignent la trajectoire dessinée par les Parlementaires d'Orléans en 2018 à savoir entre autres : rendre distincte la voix des femmes écrivaines ; affirmer qu'il existe un « écrire-ensemble » capable de renforcer les liens des écrivaines où qu'elles se trouvent; travailler à faire reconnaître la place de l'écrivaine dans son pays et réaffirmer son rôle dans le dialogue civilisationnel ; constituer un trait d'union entre le Nord et le Sud et faire circuler les idées et les auteures. Puisque les écrivains promeuvent la liberté à travers l'imaginaire, ils autorisent à fantasmer sur un monde universel plus juste. Bien que voix minoritaires et sexes victimes des violences de toutes sortes, les écrivaines sont particulièrement concernées par ce projet visant le renouvellement de la vie par le biais de l'écriture. Ce faisant, elles s'offrent un espace consensuel pour flétrir tout ce qui porte atteinte à la dignité féminine ou à l'éthique du vivre ensemble en prenant la parole pour faire entendre leurs points de vue sur les débats qui agitent notre monde : l'alliance prénatale ou le mariage pré-arrangé en fait partie. La parole féminine travaille de ce point de vue dans la perspective de s'opposer à la guerre, état de nature et vision essentialiste des liens humains, parce qu'elle témoigne du rejet de l'autre. Cette parole propose une dynamique alternative fondée sur l'idée de la paix, état de culture et vision constructionniste des liens sociaux, aux fins de postuler un seul idéal: renouveler la vie au profit de tous les hommes de la terre. 


\section{Références}

ADIAFFI, Anne-Marie, (1984), Une vie hypothéquée, NEA, Abidjan.

BEAUVOIR, Simone de, (1949), Le deuxième sexe, Gallimard, Paris.

BERNABE, Jean, CHAMOISEAU, Patrick, CONFIANT, Raphaël, (1989), Eloge de la créolité, Gallimard, Paris.

BEYALA, Calixthe, (1995), Lettre d'une Africaine à ses sœurs occidentales, Spengler, Paris.

BONONO, Angéline Solange, (2007), Le journal intime d'une épouse, SOPECAM, Yaoundé.

BOUCHENNI, Nadia, « Féministes du monde francophone, unissez-vous ! 1500 chercheuses et militantes au $8^{\text {ème }}$ CIRFF à Nanterre » mis à jour le 30. 8. 2018, https://information.tv5monde.com/terriennes/feministes-du-mondefrancophone-unissez-vous-1500-chercheuses-et-militantes-au-8eme-cirff, consulté le 31 août 2019.

BRUNEL, Pierre, PICHOIS, Claude, ROUSSEAU, André-Marie, (1983), Qu'est-ce que la littérature comparée ?, Armand Colin, Paris.

BUTLER, Judith, (2005), [1972], Trouble dans le genre: le féminisme et la subversion de l'identité., trad. Kraus Cynthia, Paris, La Découverte.

CHAULET ACHOUR, Christiane, MOULIN-CIVIL, Françoise, (2010), Le féminin des écrivaines Suds et périphéries, Encrage, Cergy-Pontoise.

DANTICAT, Edwige, (2004), Le briseur de rosée, Grasset, Paris.

DUBOIS, Jacques, (1987), "Sociocritique », DELACROIX, Maurice, HALLYN, Ferdinand, Introduction aux méthodes critiques. Méthodes du texte, Duculot, Paris, p. 290-298.

ESCARPIT, Robert, (1958), Sociologie de la littérature, PUF, Paris.

GORDIMER, Nadine, (1998), L'écriture et l'existence, 10/18, Paris.

GRESENGUET, Anne Thérèse, (2013), «Aspects sociologiques de l'écriture féminine en Afrique noire francophone ", Heuristique, Vol. 1, $\mathrm{N}^{\circ} 2$, CLE, Yaoundé, p. 47-57.

HERZBERGER FOFANA, Pierrette, (2000), Littérature féminine francophone d'Afrique noire, L'Harmattan, Paris.

HOUELLEBECQ, Michel, (2001), Plateforme, L'Harmattan, Paris. 
DE LA TEXTURE DES DIÉGĖSES DES ÉCRIVAINES DE LA POSTCOLONIE À LA CONTEXTURE D'UNE MONDIALISATION ALTERNATIVE

KRAUS, Cynthia, (2003), " "avarice épistémique" et économie de la connaissance: le pas rien du constructionnisme social ", ROUCH, Hélène, DORLIN, Elsa, FOUGEYROLLAS-SCHWEBEL, Dominique, Le corps, entre sexe et genre, L'Harmattan, Paris, p. 39-57.

LECLERC, Annie, (1974), Parole de femme, Bernard Grasset, Paris.

LECLERC, Annie, (1985), Hommes et femmes, Bernard Grasset, Paris.

MAKWARD, Christiane, COTTENET-HAGE, Madeleine, (1996), Dictionnaire littéraire des femmes de langue française: de Marie de France à Marie Ndiaye, Karthala, Paris.

MATIP, Marie-Claire, (1958), Ngonda, Bibliothèque du jeune Africain, Paris.

METTE, Isabelle, (2005), « La détérioration des “termes de la chance " : échange inégal et littérature ", Notre Librairie, $\mathrm{N}^{\circ} 157$, Dumas-Titoulet, Saint-Etienne, p. 28-35.

MITTERAND, Henri, (1980), Le discours du roman, PUF, Paris.

NAUDIER, Delphine, ROLLET, Brigitte (2007), Genre et légitimité culturelle. Quelle reconnaissance pour les femmes ?, L'Harmattan, Paris.

PIEGAY-GROS, Nathalie, (1996), Introduction à l'intertextualité, Dunod, Paris.

SEPAUSY DE, Victor « Le Parlement

https://www.actualitte.com/article/monde-edition/orleans-accueillera-leparlement-des-ecrivaines-francophones/87904

SOW FALL, Aminata, (1983), " du pilon à la machine ", Notre librairie, $\mathrm{n}^{\circ} 68$, Saint Etienne, Dumas-Titoulet Imprimeurs, Paris, p. 65-73.

STENDHAL, (1989), Le rouge et le noir, Bordas, Paris.

WABERI, Abdoulaye, (1998), "Les enfants de la postcolonie: esquisse d'une nouvelle génération d'écrivains francophones d'Afrique noire ", Notre Librairie, $n^{\circ} 135$, Dumas-Titoulet, Saint-Etienne, p. 8-15.

WARREN, Austin, WELLECK, René, (1971), La théorie littéraire, Seuil, Paris.

Recebido em 30 de agosto de 2019.

Aceito em 06 de setembro de 2019. 